This item was submitted to Loughborough's Research Repository by the author.

Items in Figshare are protected by copyright, with all rights reserved, unless otherwise indicated.

\title{
Assignment of duplicate storage locations in distribution centres to minimise walking distance in order picking
}

\section{PLEASE CITE THE PUBLISHED VERSION}

https://doi.org/10.1080/00207543.2020.1766714

\section{PUBLISHER}

Taylor and Francis

\section{VERSION}

AM (Accepted Manuscript)

\section{PUBLISHER STATEMENT}

This is an Accepted Manuscript of an article published by Taylor \& Francis in International Journal of Production Research on 28 May 2020, available online: https://doi.org/10.1080/00207543.2020.1766714

\section{LICENCE}

CC BY-NC-ND 4.0

\section{REPOSITORY RECORD}

Jiang, W, Jiyin Liu, Y Dong, and L Wang. 2020. "Assignment of Duplicate Storage Locations in Distribution Centres to Minimise Walking Distance in Order Picking". Loughborough University. https://hdl.handle.net/2134/12524495.v1. 


\title{
Assignment of duplicate storage locations in distribution centres to minimise walking distance in order picking
}

\author{
Wei Jiang ${ }^{a *}$ \\ ${ }^{a}$ Key Laboratory of Unban Rail Transit Intelligent Operation and Maintenance Technology \& \\ Equipment of Zhejiang Province, Zhejiang Normal University, Zhejiang, 321005, China \\ Jiyin Liu ${ }^{b^{*}}$ \\ ${ }^{b}$ School of Business and Economics, Loughborough University, Leicestershire, LE11 3TU, UK \\ Yun Dong ${ }^{c}$ \\ ${ }^{c}$ Key Laboratory of Data Analytics and Optimization for Smart Industry (Northeastern University), \\ Ministry of Education, Shenyang, Liaoning 110004, China \\ $\operatorname{Li~Wang}^{d}$ \\ ${ }^{d}$ School of Electronic and Information Engineering, University of Science and Technology Liaoning, \\ Anshan, Liaoning 114051, China
}

\begin{abstract}
With the rapid development of e-commerce, the orders processed in $\mathrm{B} 2 \mathrm{C}$ warehouses are characterized by heterogeneous and small volume. The traditional storage assignment strategies used in the picker-to-parts warehouses do not have advantage anymore. In this case, the scattered storage strategy is a good alternative. In this paper, we study a new scattered storage strategy which allows the same product to be placed in multiple storage locations. The correlation between products which reflects how frequently any two products will be ordered together in the same order is considered. The problem is formulated as a 0-1 integer programming model to minimize the weighted sum of distances between the products, with the weight being the elements of the correlation matrix. In order to solve large-scale problems, a GA and a basic PSO algorithm are developed. To improve solution quality, a new PSO algorithm based on the problem characteristic is designed and a hybrid algorithm combing it with GA is proposed. Experiments show that the solutions of these algorithms are close to the optimal solutions for the small-sale problems. For larger problems, the specially designed new PSO greatly improves solution quality as compared to the basic algorithms and the hybrid algorithm makes further improvement.
\end{abstract}

Keywords: Storage location assignment, Scattered storage strategy, Integer programming models, Genetic algorithm, Particle swarm optimization

\footnotetext{
*Corresponding authors. Email: jiangwei@zjnu.edu.cn; j.y.liu@lboro.ac.uk
} 


\section{Introduction}

In operations management of distribution centres, storage location assignment and order picking are two key decision problems, whereas the strategy of storage location assignment has great impacts on the efficiency of order picking (Ruben and Jacobs 1999; Bahrami, Aghezzaf, and Limere 2019; Calzavara et al. 2019). An effective storage location assignment strategy can speed up the process of order picking (Bozer and Kile 2008; Fibrianto and Hong 2019). In the last few decades, storage location assignment problem has been intensively investigated in the traditional picker-to-parts warehouses. Therefore, there has been a lot of literature over the past decades. The details can be seen in the recent survey papers (de Koster Le-Duc, and Roodbergen 2007; Roodbergen and Vis 2009; Gu, Goetschalckx, and McGinnis 2010). Several frequently used storage assignment strategies are described by de Koster et al. (2007). Random storage assigns stock keeping units (SKUs) to empty locations in the warehouse randomly (Il-Choe and Sharp 2014). For closest open location storage, the SKUs are assigned to the empty location that is closest to the picker (Hausman, Schwarz, and Graves 1976). Dedicated storage assigns SKUs to the reserved fixed locations (De Koster and Neuteboom 2001). Full-turnover storage assigns SKUs according to their turnover, and SKUs with higher volume of sales are assigned to the more conveniently accessible locations ( $\mathrm{Yu}$ and de Koster 2013). For class-based storage, incoming SKUs are divided into different classes, and then a dedicated area of the warehouse is assigned to each class (Chan and Chan 2011). For shared storage, incoming SKUs can be assigned to any suitable available area (Chen, Langevin, and Riopel 2010). In the above literature, products are stored in the traditional picker-to-parts warehouses in the form of SKU, and the same products are placed together. A main consideration of doing so is for the order pickers to easily remember the location of each product. The major drawback is the unproductive picker walking when moving from shelf to shelf and back to the central depot (Boysen, de Koster, and Weidinger 2019).

With the rapid development of e-commerce, online shopping becomes increasingly popular and, for many people, the main shopping channel. Apart from the online shopping giants such as Amazon, most department stores and supermarkets also provide online shopping options. This has led to great expansion of distribution centre and delivery operations. For such a business-to-consumer (B2C) e-commerce environment, Boysen, de Koster, and Weidinger (2019) describe its four main features. They are small order (very 
few items in each demanding order), large assortment (a wide variety of products), varying workloads and tight delivery schedules. In this case, distribution centres need to handle a huge number of very small and heterogeneous orders. Modern B2C warehouses are all equipped with advanced information technology. Handheld electronic devices are commonly used indicating the locations of products to be picked up, and so there is no need for order pickers to remember product locations. Therefore, the same products do not need to be all placed together. This is very different from the traditional warehouses and distribution centres, and so different storage allocation strategies are needed. Weidinger and Boysen (2018) first proposed the scattered storage (also denoted as mixed shelves storage) strategy. Different from the traditional storage strategies where unit loads of the same SKU are stored together, incoming batch of each SKU is broken down into individual items which may be stored all in different shelves of the warehouse, that is, the same products can be scattered in multiple positions of the warehouse. The scatterness of items can reduce the unproductive walking time of pickers, especially for trips of picking multiple products, because after picking up an tem the next one can always be found nearby. In practical distribution centres of big online retailers like Amazon, scattered storage strategy is beginning to be used and has received considerable attention recently (Weidinger and Boysen 2018).

There are few studies on the scattered storage strategy. As mentioned above, Weidinger and Boysen (2018) first proposed it to minimize the maximum distance of SKUs to some measuring points. Experiments showed that the scatterness of items can reduce unproductive picker travel. But the correlations among items, which reflect how they are frequently ordered together, were not considered. Under the scattered storage strategy, the picker routing problem was investigated (Weidinger 2018; Weidinger, Boysen, and Schneider 2019). In addition, a similar scattered storage strategy was used in Kiva mobile fulfilment system. It is an innovative warehouse automation system where robots carry shelves with products to worker stations rather than pickers take products from shelves to depots. Guan and Li (2018) proposed the scattered storage strategy based on association rules in Kiva mobile fulfilment system. Experiments showed that it can significantly decrease the number of pods to be moved. But the moving distance was not considered. Xiang, Liu, and Miao (2018) investigated the storage assignment problem where items of an SKU can be stored in multiple inventory bins and the order batching problem in the Kiva mobile fulfilment system. 
Different from the existing scattered storage strategies used in modern B2C warehouses where pickers take products from shelves in order picking, we first propose a new scattered storage strategy considering the correlation between products. As stated by Frazelle and Sharp (1989), the two products with higher correlation will often appear on the same order, storing several items frequently ordered in one order nearby can reduce the order picking time. With the continuous development of big data technology, the correlation between products can be estimated by counting a large number of historical orders. Furthermore, it is well known that minimizing the total travel distance or time in order picking is the most frequently considered objective (Weidinger 2018; de Koster, Le-Duc, and Roodbergen 2007). Therefore, we consider the scattered storage assignment problem with an objective of minimizing the sum of weighted distances of all product pairs. The distance between a pair of products is defined as the shortest among the distances between any location of one of these two products and any location of the other product, and the weight is set as the correlation of the two products. This objective can help reduce the total travel distance in order picking.

Because the storage location assignment problem is NP-hard (Frazelle and Sharp 1989), researchers were devoted to developing different approximate algorithms. Bottani et al. (2012) used GA to determine the storage locations to reduce the distance of picking. Pan et al. (2015) developed a genetic based heuristic to solve storage assignment problem for a pick-and-pass system. Yang et al. (2017) used an improved PSO algorithm for intelligent warehouses. Kuo et al. (2016) developed a modified PSO and GA algorithm for solving the item assignment problem in synchronized zoning system. Zhao et al. (2015) developed two optimization approaches based on PSO and GA for the storage allocation problem of outbound containers. In addition to GA and PSO, other heuristics such as simulated annealing, variable neighborhood search, tabu search and differential evolution algorithm were also used in integrated or similar problems (Muppani and Adil 2008; Yang et al. 2015; Chen, Langevin, and Riopel 2010; Xiao and Zheng 2010; Pang and Chan 2017; Calzavara et al. 2019; Tang, Sun, and Liu 2016; Tang, Li, and Chen 2019; Tang, Dong, and Liu 2015). We will use the two most widely used methods GA and PSO to solve our problem.

The remainder of the paper is organized as follows. Section 2 gives a more detailed description of the new storage policy that each product can be assigned to multiple storage locations. A genetic algorithm, a basic PSO algorithm, a new PSO algorithm based on the problem characteristic as well as a hybrid algorithm combining GA and the new PSO are 
then proposed in Section 3. Section 4 reports the results of the experiments conducted to evaluate these algorithms. Finally, Section 5 concludes the paper.

\section{Problem description and the mathematical model}

\subsection{Problem description}

The problem we study is to assign storage space in a distribution centre to a given number of products. The storage area is composed of basic storage locations. The number of locations is more than the number of products. Each location can only be used to store (pieces of) one product. Each product must be stored in at least one location while it can be stored in multiple storage locations. The layout of the storage area is given and thus the distance between any two locations can be calculated. For any two products, there is a correlation which reflects the probability of them being ordered together. The correlations can be calculated from historical orders. The scattered storage assignment problem in this paper is to determine the storage locations for each product so that the weighted sum of distances of all product pairs is minimized. Here the distance between a pair of products A and $\mathrm{B}$ is defined as the shortest among the distances between any location of product $\mathrm{A}$ and any location of product $\mathrm{B}$, and the weight is set as the correlation of the two products. The objective is chosen to help reducing the estimated travel distance in the order picking operation. Fig. 1 shows a small-scale example where eight products numbered as 1 to 8 are stored in the warehouse with sixteen storage locations. The assignment shown in the figure is only one of the feasible assignments. We want to find an optimal assignment with minimum weighted distances of all pairs of these eight products.

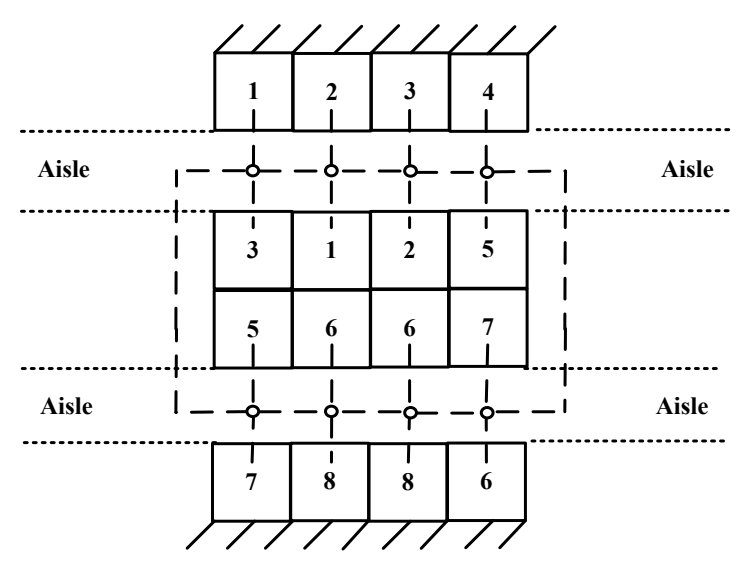

Fig.1 An example of the scattered storage assignment problem 


\subsection{The mathematical model}

\subsubsection{Parameters:}

$G \quad-$ The total number of products.

$L \quad-\quad$ The total number of storage locations.

$K \quad-$ Maximum allowable number of storage locations for each product.

$M \quad-$ A sufficiently large number.

$i, j \quad-\quad$ Indexes for products, and $1 \leq i, j \leq G$.

$l, m$ - Indexes for locations and $1 \leq l, m \leq L$.

$r_{i j}-$ Correlation of products $i$ and $j . r_{i j}$ is equal to the total number of orders including products $i$ and $j$ simultaneously divided by the total number of historical orders used for statistics.

$d_{l m}-$ The distance between location $l$ and location $m$.

\subsubsection{Decision variables:}

$x_{i l}= \begin{cases}1 & \text { if product } i \text { is assigned to location } l \\ 0 & \text { otherwise }\end{cases}$

$y_{i j l m}= \begin{cases}1 & \begin{array}{l}\text { if product } i \text { is in locaiton } l, \text { product } j \text { is in locaiton } m, \text { and the distance } \\ \text { between } l \text { and } m \text { is the shortest among products } i \text { and } j\end{array} \\ 0 & \text { otherwise }\end{cases}$

\subsubsection{Model:}

With the above notation, the mathematical model denoted by SSS can be formulated as follows:

(SSS) $\quad \min \sum_{i=1}^{G} \sum_{\substack{j=1 \\ j \neq i}}^{G} \sum_{l=1}^{L} \sum_{m=1}^{L} r_{i j} \cdot d_{l m} \cdot y_{i j l m}$

s.t.

$\sum_{i=1}^{G} x_{i l}=1 \quad \forall l=1, \cdots, L ;$

$\sum_{l=1}^{L} x_{i l} \geq 1 \quad \forall i=1, \cdots, G$;

$\sum_{l=1}^{L} x_{i l} \leq K \quad \forall i=1, \cdots, G$;

$y_{i j l m} \leq x_{i l} \quad \forall i, j=1, \cdots, G$, and $j \neq i, \forall l, m=1, \cdots, L ;$ 


$$
\begin{array}{ll}
y_{i j l m} \leq x_{j m} & \forall i, j=1, \cdots, G, \text { and } j \neq i, \forall l, m=1, \cdots, L ; \\
\sum_{l=1}^{L} \sum_{m=1}^{L} y_{i j l m}=1 & \forall i, j=1, \cdots, G, \text { and } j \neq i ; \\
x_{i l} \in\{0,1\} & \forall i=1, \cdots, G, \forall l=1, \cdots, L ; \\
y_{i j l m} \in\{0,1\} & \forall i, j=1, \cdots, G \text {, and } j \neq i, \forall l, m=1, \cdots, L ;
\end{array}
$$

In this model, objective (1) is to minimize the sum of weighted distances of all product pairs in order to reduce the total travel distance in order picking cycle. It is noted that if $y_{i j l m}=1$, distance $d_{l m}$ is the minimum distance among all distances between the pair of products $i$ and $j$ according to the definition of $y_{i j l m}$. The weight is the correlation of the two products, which represents how frequently the two products will be ordered at the same time. Constraints (2) ensure that each location can only be used to store one product. Constraints (3) and (4) ensure that each product can be placed in at least one and at most $K$ locations. Constraints (5) and (6) restrict that $y_{i j l m}$ can only be equal to 1 when both $x_{i l}$ and $x_{j m}$ are equal to 1 . Constraints (7) state that among all the distances between a pair of products $i$ and $j$, only one is the shortest. Finally, constraints (8-9) define the types and ranges of the decision variables.

To compare the difference between the scattered storage strategy and the traditional storage strategy where each product is stored in a continuous area (locations next to each other), a corresponding traditional storage model denoted as $\mathrm{SSS}_{\mathrm{T}}$ is built by adding constraints (10) model SSS. Note that the indexes of the storage locations are sequentially numbered as shown in Fig.9 of Section 4.

$$
K \cdot x_{i l}+\sum_{l^{\prime}=l+2}^{L} x_{i l^{\prime}} \leq K+K \cdot x_{i, l+1} \quad \forall i=1, \cdots, G ; \forall l=1, \cdots, L-2 ;
$$

Constraints (10) ensure that if product $i$ is stored in location $l$ and any location from $l+2$ to $L$, then it must also be stored in location $l+1$. The constraints ensure that the same products are continuously stored in the warehouse.

\subsubsection{A heuristic rule of fixing $K_{i}$ :}

Tests show that model SSS takes long even for small-scale problems. The maximum number of storage locations allowed for each product $K$ can be large which makes the solution space very large. However, the total number of storage locations is limited. If we can fix the number of locations $K_{i}$ for each product $i$, the solution space will be much smaller and the model may be solved more efficiently. Of course, each $K_{i}$ needs to be 
carefully chosen to keep a good solution quality.

It is important to note that if products $i$ and $j$ are frequently ordered together, the value of $r_{i j}$ (correlation of products $i$ and $j$ ) is higher, otherwise it is lower. The greater the sum of product $i$ 's correlations with other products, the greater the number of storage locations for product $i$ should be, in order for it to be stored close to each of the other products highly correlated to it. Based on this idea, we propose the following method to fix $K_{i}$.

Step 1: Compute $\hat{r}_{i}=\sum_{j=1}^{G} r_{i j}$ for each product $i$, and then let $R_{i}=\frac{\hat{r}_{i}}{\sum_{i=1}^{G} \hat{r}_{i}} \times L$. Clearly, $\sum_{i=1}^{G} R_{i}=L . R_{i}$ indicates how many of the $L$ locations should be assigned to product $i$.

Step 2: For each product $i$, initialize $K_{i}=1$ to guarantee that each product will be stored in at least one location. Now the number of locations already allocated is $L_{a}=G$.

Step 3: Find product $i^{*}$ with the maximum value of $R_{i^{*}}$. Let $K_{i^{*}}=K_{i^{*}}+1, R_{i^{*}}=R_{i^{*}}-1, L_{a}=$ $L_{a}+1$.

Step 4: If $L_{a} \geq L$, stop; otherwise, go to Step 3 .

By fixing $K_{i}$, constraints (3) and (4) in model SSS can be replaced by constraints (11).

$$
\sum_{l=1}^{L} x_{i l}=K_{i} \quad \forall i=1, \cdots, G
$$

With this change, the new model will be referred to as $\operatorname{SSS}^{K_{i}}$. Note that with fixed $K_{i}$, the optimal solution of the new model $\mathrm{SSS}^{K_{i}}$ is not guaranteed be optimal for the original problem. However, it gives the same solutions with model SSS for small-scale problems tested in Section 4. The solution time of model $\operatorname{SSS}^{K_{i}}$ is much shorter and it can solve problems with larger scale. Therefore, fixing $K_{i}$ is a good method for solving our problem. With problem scale further increases, the proposed models cannot be solved within acceptable time. Therefore, we design several intelligent heuristic algorithms based on the rule of fixing $K_{i}$.

\section{Genetic algorithm and Particle swarm optimization algorithm}

Because the proposed model can only get optimal solutions for small-scale problems 
within acceptable time, GA and PSO are proposed to get approximate optimal solutions for medium and large-scale problems.

\subsection{Genetic algorithm}

It can be seen from the algorithm review on the storage location assignment problem in Section 1, GA is widely used to solve this type of problems (Bottani et al. 2012; Pan et al. 2015; Kuo et al. 2016; Zhao et al. 2015), thus we adopt GA to solve our problem at first.

\subsubsection{Encoding:}

The values of the model variables $x_{i l}$, corresponds naturally to a two-dimensional binary coding scheme where a row represents a product and a column represents a location. In GA we use a more compact one-dimensional encoding scheme where each bit represents a location and the number in a bit represents the product stored in this location. The two schemes can be converted to each other easily as shown in Fig.2.

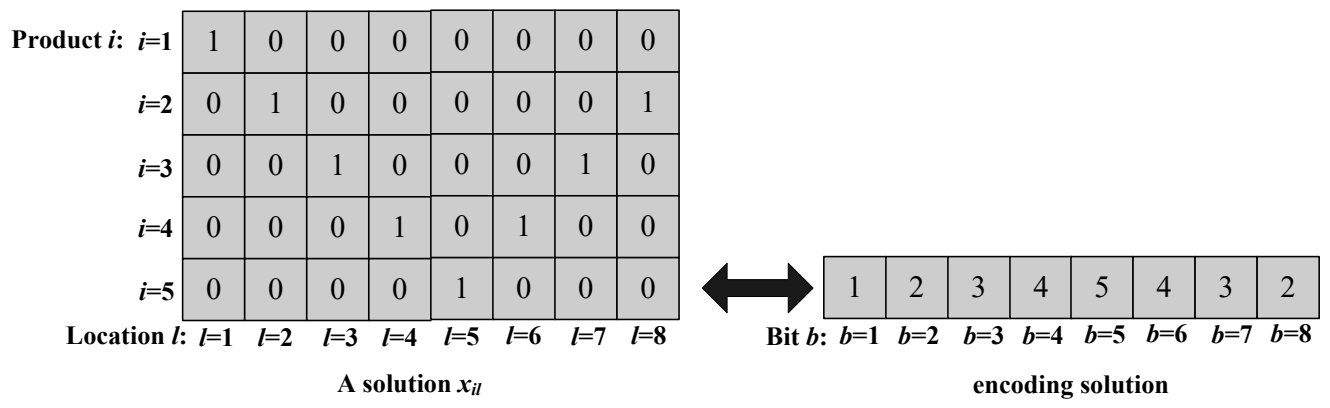

Fig. 2 The relation between model solution and GA encoding solution

\subsubsection{Initial solutions}

We generate each individual in the initial population randomly in the following way. For each bit of the individual, from bit 1 to bit $L$, randomly select a product and let the bit to be the index of this product. When a product has already been selected to $K_{i}$ bits, it cannot be selected anymore for the remaining bits.

\subsubsection{Fitness function:}

The objective function of this problem is to be minimized. It needs to be converted to a fitness function which is to be maximized in GA. For any population, we first calculate a relatively large value $F=$ (the maximum objective value - the minimum objective value) + the maximum objective value. Then, the fitness value of any individual is calculated as $F$ minus its objective value. In this way, a lower objective value corresponds to a higher fitness value. 


\subsubsection{Selection}

Roulette wheel selection is adopted. The probability of each individual being selected is proportional to its fitness value. Given population size $S$ and fitness value $f_{S}$ of each individual $s, s=1, \ldots, S$, the probability for $s$ being selected is:

$$
p_{s}=\frac{f_{s}}{\sum_{s=1}^{S} f_{s}}
$$

\subsubsection{Crossover}

Crossover operates on two selected individuals. The commonly used two-point crossover is adopted here. As shown in Fig.3, two bits Bit1 and Bit2 in individuals 1 and 2 are randomly chosen, and then their segments between Bit1 and Bit2 are exchanged to produce new individuals 1 ' and 2 ' of the next generation.

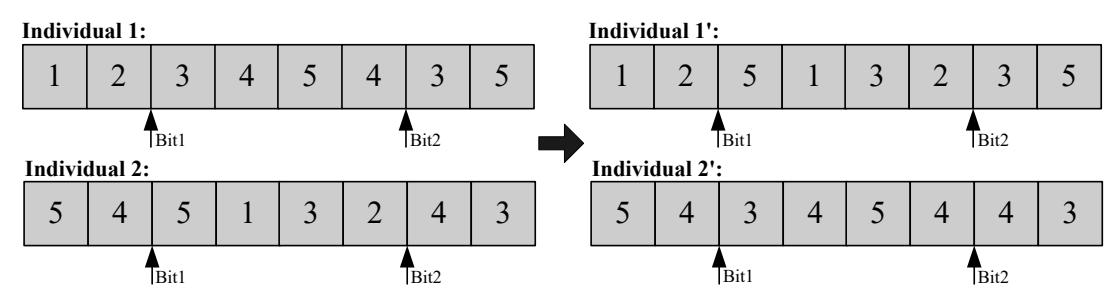

Fig. 3 The process of crossover

\section{Feasibility correction:}

Any feasible individual has to satisfy the condition that the number of bits occupied by product $i$ must be equal to $K_{i}$. After exchanging bits in the crossover, the resulting new individuals may be infeasible. We use a sub-procedure ReviseFeasible $(s)$ to make an infeasible individual feasible.

\section{ReviseFeasible(s):}

First check individual $s$. For product $i$, if the number of its appearances in the individual is more than $K_{i}$, we say that there are redundant copies of this product; if the number of its appearances is less than $K_{i}$, we say that there are absent copies of this product. In this way we can obtain a set of redundant copies of products, redundant products in short, and similarly a set of absent products. The feasibility correction is then done by replacing the redundant products with the absent products. For each redundant product in the set, randomly select one among all the bits in $s$ storing the same product and change this bit to a product randomly chosen from the absent product set. Remove this redundant product and 
this absent product from the relevant sets and repeat this until the sets become empty. Fig. 4 shows an example where there are 5 products and 8 positions. The value of each $K_{i}$ $(i=1, \ldots, 5)$ is given in the figure. Individual $s$ is infeasible with the redundant product set $=\{1,1,4\}$ and absent product $\operatorname{set}=\{2,2,3\}$. Applying the above correction, it becomes feasible individual $s^{\prime}$.

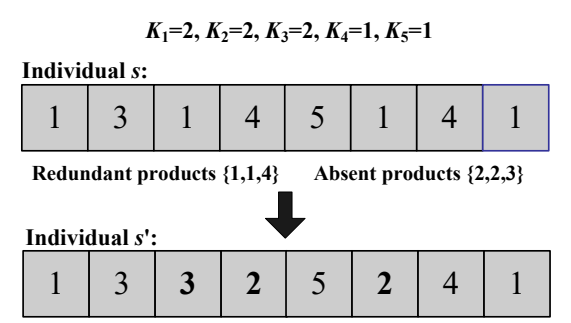

Fig.4 The process of feasibility correction

\subsubsection{Mutation}

The mutation operation randomly selects two bits in the individual and swaps their values. Fig. 5 is an example where the values of the second and fifth bits are swapped.

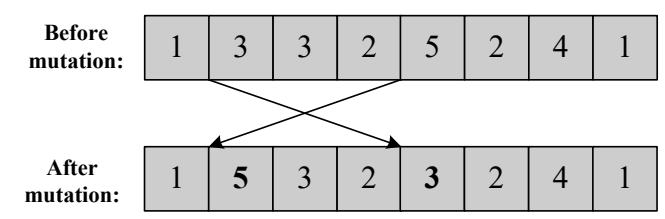

Fig.5 The process of mutation

Because the mutation does not change the number of storage locations for each product, it does not affect feasibility.

\subsubsection{The best solution saving strategy}

In each generation, the minimum objective value in the current generation is compared with that in the previous generation. If the minimum objective value in the previous generation is better, the best individual in the previous generation is added into the current population replacing an individual randomly chosen.

\subsection{Particle swarm optimization (PSO) algorithm}

Particle swarm optimization (PSO) algorithm is also a common algorithm used to solve the storage location assignment problem (Yang et al. 2017; Kuo et al. 2016; Zhao et al. 2015). Therefore, we use PSO to solve our problem and compare the performance with GA.

\subsubsection{Basic particle swarm optimization algorithm}


First, we use the basic PSO to solve our problem. Its main framework is presented step by step below.

Step 1: Set the iteration number $n=1$. Initialize a swarm of particles (particle positions). The product located in each bit for a particle is generated in the same way as the initial solution of GA, and its corresponding velocity is an integer randomly generated from $[-G, G]$.

Step 2: For each particle in the current swarm, calculate objective value $\sum_{\substack{i=1 \\ \text { G }}}^{G} \sum_{\substack{j \neq i \\ j \neq i}}^{G} r_{i j} D_{i j}$, where $D_{i j}$ is the shortest distance between products $i$ and $j$ in the solution.

Step 3: Update the best position of particle $s, \mathrm{pBest}_{s}^{n}=\left[\mathrm{pBest}_{s 1}^{n} \mathrm{pBest}_{s 2}^{n}, \ldots, \mathrm{pBest}_{s L}^{n}\right]$, and the best position of the swarm, $\mathrm{gBest}^{n}=\left[\mathrm{gBest}_{1}^{n}, \mathrm{gBest}_{2}^{n}, \ldots, \mathrm{gBest}_{L}^{n}\right]$.

Step 4: Update the velocity for each particle according to Formula (13), and update each particle according to Formula (14).

Step 5: The values of some bits in the particle obtained above may not be integer. Round off these to integers. If the value in any bit is below 1 or above $G$, change it to 1 or $G$ accordingly. Then if a particle is infeasible, repair it with ReviseFeasible(s) as shown in subsection 3.1.5.

Step 6: If the iteration number reaches the preset maximum value, stop; otherwise, $n=n+$ 1 and go to Step 2.

(13) and (14) are the velocity and position updating formulas, respectively.

$$
\begin{aligned}
& V_{s b}^{n+1}=w V_{s b}^{n}+c_{1} r_{1}\left(\mathrm{pBest}_{s b}^{n}-X_{s b}^{n}\right)+c_{2} r_{2}\left(\mathrm{gBest}_{b}^{n}-X_{s b}^{n}\right), b=1, \ldots, L \\
& X_{s b}^{n+1}=X_{s b}^{n}+V_{s b}^{n+1}, b=1, \ldots, L
\end{aligned}
$$

Where $w$ is inertia coefficient, $c_{1}$ and $c_{2}$ are acceleration coefficients, $r_{1}$ and $r_{2}$ are random numbers between zero and one. $V_{s b}^{n}$ and $X_{s b}^{n}$ are the velocity and position (encoding) in bit $b$ for particle $s$.

Fig.6 is an example showing the transformation of a particle in an iteration of the PSO algorithm. The left part of Fig.6 shows the current position of the particle, its best position and the best position of all particles up to this iteration. The first bits of these three solutions are products 4, 3 and 3, respectively. Using (13) and (14), the product in the first bit of the particle is updated to 2. The updating process for each bit of the particle step by 
step can be seen in the middle part of Fig.6, with the last row being the result. This result is an infeasible particle, so it is then revised to a feasible one that is shown in the right part of Fig.6.

\begin{tabular}{|c|c|c|c|c|c|c|c|c|c|c|c|c|c|c|c|c|c|c|c|c|c|c|c|}
\hline \multirow{2}{*}{\multicolumn{8}{|c|}{ pBest: }} & \multicolumn{8}{|c|}{ Update the product in each position step by step: } & & & & & & & & \\
\hline & & & & & & & & 2 & 2 & 3 & 1 & 1 & 3 & 5 & 2 & & & & & & & & \\
\hline$b=1$ & $\frac{2}{b=2}$ & $b=3$ & $\frac{2}{b=4}$ & $\frac{1}{b=5}$ & $\frac{3}{b=6}$ & & & 2 & 1 & 3 & 1 & 1 & 3 & 5 & 2 & & & & & & & & \\
\hline Cur & ent & parti & cle: & & & & & 2 & 1 & 5 & 1 & 1 & 3 & 5 & 2 & Revi & e to & fe & ibl & pat & icle: & & \\
\hline 4 & 2 & 3 & 1 & 1 & 3 & 5 & 2 & \begin{tabular}{|l|}
2 \\
\end{tabular} & 1 & 5 & 2 & 1 & 3 & 5 & 2 & 2 & 1 & 5 & 2 & 4 & 3 & 1 & 3 \\
\hline & $b=2$ & $b=3$ & $b=4$ & $b=5$ & $b=6$ & $b=7$ & $\overrightarrow{b=8}$ & \begin{tabular}{|l|} 
\\
2
\end{tabular} & 1 & 5 & $\frac{2}{2}$ & 1 & 3 & 5 & $\frac{2}{2}$ & & & $b=3$ & $p=4$ & $b=5$ & & $b=7$ & \\
\hline$\frac{\mathrm{gBe}}{3}$ & & & & & $?$ & & 2 & 2 & 1 & 5 & 2 & 1 & 3 & 5 & 2 & & & & & & & & \\
\hline & $\frac{1}{b=2}$ & & & 1 & & & & 2 & 1 & 5 & 2 & 1 & 3 & 1 & 2 & & & & & & & & \\
\hline & & & & & & & & 2 & 1 & 5 & 2 & 1 & 3 & 1 & 1 & & & & & & & & \\
\hline
\end{tabular}

Fig.6 The transformation of a particle

\subsubsection{Improved particle swarm optimization algorithm}

In the basic PSO algorithm, different bits of a particle are updated independently according to (13) and (14) and the particle is not considered as a whole. This often leads to infeasibility and revision is needed. As a result, the objective value of the new particle may not be improved as intended.

To overcome this, we develop a new PSO algorithm where a particle is considered as a whole and design new velocity and position updating methods that maintain feasibility.

The overall framework of the new PSO procedure is similar to that of the basic PSO. The main difference is that step 4 is replaced with the new updating method and step 5 in the basic PSO is not needed in the new PSO.

In our new updating method, the updating of a particle is by moving the bits around within the particle, and the velocity represents the steps for the product in each bit to move.

The basic idea for determining the velocity (moving steps) $V_{s b}^{n+1}$ for a bit $b$ of the current particle $X_{s}^{n}$ is to move the product in this bit towards the corresponding bits of the same product in the best position of this particle pBest $_{s}^{n}$ and in the global best position of all particles gBest $^{n}$. We denote these corresponding bits as $b^{\prime}$ and $b^{\prime \prime}$, respectively. Note that the same product $i$ is stored in $K_{i}$ bits in a particle. If bit $b$ is the $k^{\text {th }}$ bit (from left to right) that stores this product, then $b^{\prime}$ and $b^{\prime \prime}$ must be the $k^{\text {th }}$ bit storing this product correspondingly in $\operatorname{pBest}_{s}^{n}$ and gBest $^{n}$. We introduce an operator $\Theta$ to calculate bit differences so that $\left(\mathrm{pBest}_{s b^{\prime}}^{n} \ominus X_{s b}^{n}\right)$ represents the process of finding the bit $b^{\prime}$ in $\mathrm{pBest}_{s}^{n}$ 
corresponding to bit $b$ in $X_{s}^{n}$, and take the difference $\left(b^{\prime}-b\right)$. Similarly, $\left(\operatorname{gBest}_{b^{n}}^{n} \ominus X_{s b}^{n}\right)$ gets the difference $\left(b^{\prime \prime}-b\right)$. These differences are the numbers of steps that the product needs to move in order to reach to the corresponding bits in the two best particle positions. We set the velocity for the bit as the random combination of these two differences.

$$
V_{s b}^{n+1}=r_{1}\left(\mathrm{pBest}_{s b^{\prime}}^{n} \ominus X_{s b}^{n}\right)+r_{2}\left(\mathrm{gBest}_{b^{n}}^{n} \ominus X_{s b}^{n}\right)
$$

Here $r_{1}$ and $r_{2}$ are random numbers between zero and one. If the resulting $V_{s b}^{n+1}$ is not integer, we round it off to the nearest integer.

We introduce another operator $\oplus$ to express the movement of one bit in the particle. $X_{s}^{n} \oplus V_{s b}^{n+1}$ means to move the product in bit $b$ of $X_{s}^{n}$ for $V_{s b}^{n+1}$ bits to bit $b+V_{s b}^{n+1}$ (the movement is to the right if $V_{s b}^{n+1}$ is positive and to the left otherwise) and at the same time to shift the products between bits $b$ and $b+V_{s b}^{n+1}$ (including $b+V_{s b}^{n+1}$ but not $b$ ) by one bit in the opposite direction. In the above description, if cases of $b+V_{s b}^{n+1}<1$ and $b+V_{s b}^{n+1}>L$, we will take $b+V_{s b}^{n+1}$ as 1 and $L$, respectively. The updating of the position of a particle is then defined as the movements of a series of randomly selected bits.

$$
X_{s}^{n+1}=X_{s}^{n} \oplus V_{s b_{1}}^{n+1} \oplus \cdots \oplus V_{s b_{L 1}}^{n+1}
$$

Here $L 1 \quad(1 \leq L 1 \leq L)$ is the number of bits moved in the updating process. $b_{1}$ is a randomly selected bit between 1 and $L$. After the movement of the product in bit $b_{1}$, the new bit where it is stored is denoted as $b_{1}^{\text {new }}$. Then if $V_{s b_{1}}^{n+1} \geq 0\left(V_{s b_{1}}^{n+1}<0\right), b_{2}$ is selected as the bit on the right (left) of $b_{1}^{\text {new }}$. If the special case of $b_{1}^{\text {new }}=L\left(b_{1}^{\text {new }}=1\right), b_{2}=1\left(b_{2}=L\right)$. Then $V_{s b_{2}}^{n+1}$ is calculated based on the partially updated particle and the product in bit $b_{2}$ is moved to a new bit $b_{2}^{\text {new }}$. In a similar way, each of the following bits $b_{i}, i=3, \ldots, b_{L 1}$, is selected based on the new bit $b_{i-1}^{\text {new }}$ to which the product in $b_{i-1}$ was moved, and $V_{s b_{i}}^{n+1}$ is calculated before the product in the bit is moved.

Fig.7 and Fig. 8 show an example updating process including movements of at least two bits, each figure shows the movements for one selected bit. In each figure, the left part shows the particle being updated and the two best particles, and the right part shows the result after the movement of one bit. In this example, $b_{1}$ is selected as 1 . 
In Fig. 7, the product in $b_{1}$ is 1 , then $b_{1}^{\prime}=3, b_{1}^{\prime \prime}=6$, thus $b_{1}^{\prime}-b_{1}=2, b_{1}^{\prime \prime}-b_{1}=5 . \quad r_{1}$ and $r_{2}$ are taken as 0.4 and 0.5 , respectively. According to $(15), V_{s b_{1}}^{n}=0.4 \times 2+0.5 \times 5=3.3$. Hence, product 2 in bit $b_{1}$ needs to move 3 bits (obtained by rounding off) to the right, that is, product 2 is moved from bit 1 to bit 4 , thus $b_{1}^{\text {new }}=4$. At the same time, products $1,3,5$ between bits 2 and 4 are shifted by one bit to the left.

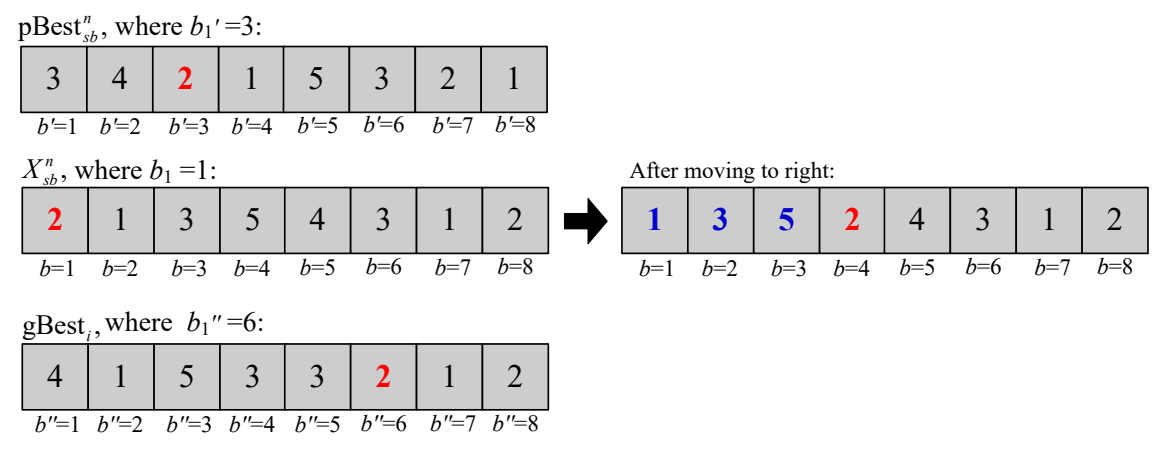

Fig.7 The process of moving to the right

In Fig.8, From $b_{1}^{\text {new }}=4$ we get $b_{2}=5, b_{2}^{\prime}=2, b_{2}^{\prime \prime}=1$, thus $b_{2}^{\prime}-b_{2}=-3, b_{2}^{\prime \prime}-b_{2}=-4$. Take $r_{1}=0.6$ and $r_{2}=0.5$, then $V_{s b_{2}}^{n}=0.6 \times(-3)+0.5 \times(-4)=-3.8$. It represents that product 4 in bit $b_{2}$ needs to move 4 bits (obtained by rounding off) to the left. So product 4 is moved from bit 5 to bit 1 , thus $b_{2}^{\text {new }}=1$. At the same time, shift products $1,3,5,2$ between bits 1 and 4 by one bit to the right. If $L 1=2$, the updating process is completed now. If $L 1>2$, because $b_{2}^{\text {new }}=1, b_{3}$ will be selected as $L=8$ and the updating process will be continued.

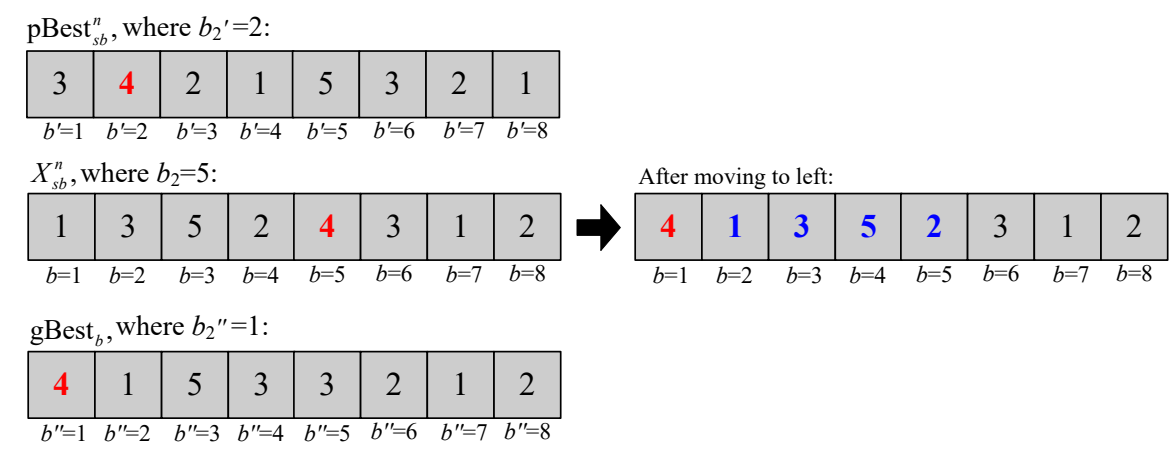

Fig. 8 The process of moving to the left

\subsubsection{A hybrid algorithm of GA and improved PSO}

Numerical test shows that GA is better in global search while PSO converges more quickly. To further improve solution quality, we propose to combine GA and the improved 
PSO to form a hybrid algorithm. While the total computation time is not increased, the algorithm first runs GA and then the population of the final generation is taken as the initial population of the improved PSO. Then, the improved PSO is performed to continue the search for better solutions. The performance of the hybrid algorithm can be seen in Section 4.

\subsubsection{An example for the hybrid algorithm}

In order to illustrate how to apply the proposed hybrid algorithm to solve the problem in this paper, a numeric example is given in this Section. For problem 5-8 (5 products and 8 storage locations) and problem 6-12, the objective value obtained by GA for each instance is roughly the same with that obtained by the hybrid algorithm. It shows that GA can obtain good solutions for the two small problems. For problem 8-16, PSO can further obtain better solutions based on the solutions obtained by GA. Therefore, we selected the numeric example with 8 products and 16 storage locations.

The following is the correlation matrix of eight products:

$$
\left(\begin{array}{llllllll}
0.00 & 5.30 & 4.21 & 3.64 & 3.27 & 2.93 & 2.59 & 2.27 \\
5.30 & 0.00 & 3.24 & 2.88 & 2.54 & 2.30 & 2.06 & 1.86 \\
4.21 & 3.24 & 0.00 & 2.44 & 2.20 & 2.00 & 1.79 & 1.63 \\
3.64 & 2.88 & 2.44 & 0.00 & 2.01 & 1.83 & 1.65 & 1.53 \\
3.27 & 2.54 & 2.20 & 2.01 & 0.00 & 1.70 & 1.56 & 1.43 \\
2.93 & 2.30 & 2.00 & 1.83 & 1.70 & 0.00 & 1.44 & 1.35 \\
2.59 & 2.06 & 1.79 & 1.65 & 1.56 & 1.44 & 0.00 & 1.27 \\
2.27 & 1.86 & 1.63 & 1.53 & 1.43 & 1.35 & 1.27 & 0.00
\end{array}\right)
$$

The following is the distance matrix of 16 positions:

$$
\left(\begin{array}{llllllllllllllll}
0 & 1 & 2 & 3 & 4 & 3 & 2 & 1 & 3 & 4 & 5 & 6 & 6 & 5 & 4 & 3 \\
1 & 0 & 1 & 2 & 3 & 2 & 1 & 2 & 4 & 5 & 6 & 5 & 5 & 6 & 5 & 4 \\
2 & 1 & 0 & 1 & 2 & 1 & 2 & 3 & 5 & 6 & 5 & 4 & 4 & 5 & 6 & 5 \\
3 & 2 & 1 & 0 & 1 & 2 & 3 & 4 & 6 & 5 & 4 & 3 & 3 & 4 & 5 & 6 \\
4 & 3 & 2 & 1 & 0 & 1 & 2 & 3 & 6 & 5 & 4 & 3 & 3 & 4 & 5 & 6 \\
3 & 2 & 1 & 2 & 1 & 0 & 1 & 2 & 5 & 6 & 5 & 4 & 4 & 5 & 6 & 5 \\
2 & 1 & 2 & 3 & 2 & 1 & 0 & 1 & 4 & 5 & 6 & 5 & 5 & 6 & 5 & 4 \\
1 & 2 & 3 & 4 & 3 & 2 & 1 & 0 & 3 & 4 & 5 & 6 & 6 & 5 & 4 & 3 \\
3 & 4 & 5 & 6 & 6 & 5 & 4 & 3 & 0 & 1 & 2 & 3 & 4 & 3 & 2 & 1 \\
4 & 5 & 6 & 5 & 5 & 6 & 5 & 4 & 1 & 0 & 1 & 2 & 3 & 2 & 1 & 2 \\
5 & 6 & 5 & 4 & 4 & 5 & 6 & 5 & 2 & 1 & 0 & 1 & 2 & 1 & 2 & 3 \\
6 & 5 & 4 & 3 & 3 & 4 & 5 & 6 & 3 & 2 & 1 & 0 & 1 & 2 & 3 & 4 \\
6 & 5 & 4 & 3 & 3 & 4 & 5 & 6 & 4 & 3 & 2 & 1 & 0 & 1 & 2 & 3 \\
5 & 6 & 5 & 4 & 4 & 5 & 6 & 5 & 3 & 2 & 1 & 2 & 1 & 0 & 1 & 2 \\
4 & 5 & 6 & 5 & 5 & 6 & 5 & 4 & 2 & 1 & 2 & 3 & 2 & 1 & 0 & 1 \\
3 & 4 & 5 & 6 & 6 & 5 & 4 & 3 & 1 & 2 & 3 & 4 & 3 & 2 & 1 & 0
\end{array}\right)
$$

The layout of the warehouse is similar to Fig.9, and the number of locations in each 
row is four. The numbers of locations for the eight products are 3, 2, 2, 2, 2, 2, 2,1 according to heuristic rule of fixing $K_{i}$, respectively. Ten initial solutions of GA randomly selected in the population (the size is 200) are as follows:

$[2,8,5,6,1,1,4,2,6,7,1,4,7,5,3,3] ; \quad[6,3,4,8,3,5,4,5,2,6,7,2,7,1,1,1]$;

$[3,7,1,6,1,6,8,1,7,3,5,4,2,2,5,4] ; \quad[1,3,1,8,5,1,3,4,5,4,7,2,2,7,6,6]$;

$[7,1,6,4,2,3,1,2,3,5,4,5,6,7,1,8] ; \quad[3,1,6,1,1,7,4,4,5,3,6,8,7,2,2,5]$;

$[1,1,3,2,3,5,4,5,2,7,6,6,7,4,8,1] ; \quad[1,8,6,5,2,4,7,2,1,5,4,3,3,7,6,1]$;

$[3,1,7,6,5,3,5,2,7,2,4,8,4,1,6,1] ; \quad[4,5,6,4,5,7,1,2,1,2,3,6,7,1,3,8]$;

The best solution obtained by GA after 250 iterations is: $[1,8,7,5,6,2,4,3,1,5,1,4$, $6,7,3,2]$, and the minimum objective value obtained by GA after 250 iterations is 83.67. The best solution obtained by GA is used as the initial solutions of PSO, and then the best solution obtained by PSO after 150 iterations is $[1,8,2,6,7,5,4,3,1,2,4,1,5,6,3,7]$, and the corresponding objective value is 80.71 . We can see that PSO has a further improvement based on GA.

\section{Computational experiments}

In this section, in order to verify the performance of the proposed models and algorithms, we carry out computational experiments on instances of different scales generated randomly. The models and algorithms are coded in $\mathrm{C}++$ language and tested on a computer with Intel Core i5 3.40GHz CPU and 8.00GB RAM. CPLEX 12.8 is used as an optimization solver to solve the models.

\subsection{Description of the tested data}

In order to generate realistic values of product correlations $r_{i j}$, we generate a large number of orders and count the number of orders where products $i$ and $j$ both appear. It is worth noting that the demands of products usually follow the ABC rule in practice. Class $\mathrm{A}$ products account for about $10 \%$ of the total number of products, but their sales volume is relatively large accounting for $70 \%-80 \%$. Class B products account for about $20 \%$, and their sales volume is moderate, accounting for $10 \%$ to $20 \%$. Class $\mathrm{C}$ products are the rest containing a large variety of products accounting for about $70 \%$, but their sales volume is small accounting for $0 \%$ to $10 \%$. We generate 1000000 orders randomly. For small-scale problems, 2-3 different products are generated on each order, while for medium and large-scale problems, 2-5 different products are generated. Each product in the order is 
generated randomly from a distribution based on the ABC rule. With the 1000000 orders, we count the total number of orders where two products $i$ and $j$ are both in, divide the number by 1000000 , and then multiply 100 to get $r_{i j}$. Multiplying 100 is to avoid the value of $r_{i j}$ being too small, but this does not affect the problem.

Given the warehouse layout, the distance between any two locations $l$ and $m, d_{l m}$, can be calculated. We set the storage area in warehouse as a rectangular area with aisles, each aisle has access to storage locations on both sides. The locations are numbered sequentially as illustrated by the example in Fig.9. The storage locations are numbered consecutively from 1 to 40 and example distances between adjacent locations are shown. In our experiments, the numbers of locations in each row are set to 4, 10 and 50 for small, medium and large-scale problems, respectively. We use "the total number of different products-the total number of storage locations" to indicate different scales of problems. For example, " $5-8$ " represents problems in which there are five different products and the total number of storage locations is eight.

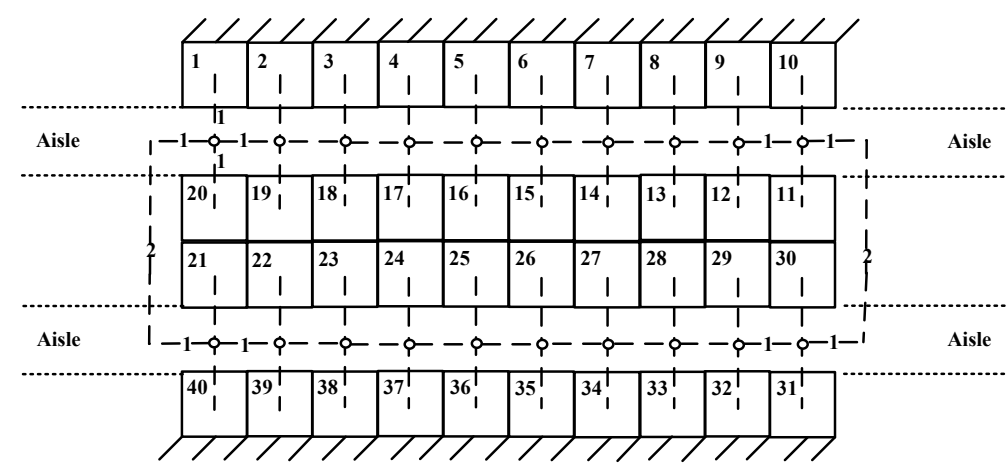

Fig.9 The layout of the warehouse's storage area

\subsection{Computational results for the different models}

In this section, we test the models proposed in Section 2.2 on four groups of small-scale problems to verify their effectiveness. For each group ten problem instances are solved, and the average objective value and CPU computation time measured in seconds are shown in Table 1. From the results, it is clear that the objective values obtained by SSS are much smaller than those obtained by $\mathrm{SSS}_{\mathrm{T}}$. This indicates that the scattered storage strategy is better than the traditional continuous storage strategy. Model SSS cannot obtain optimal solutions for 8-20. Here the values of $K$ for these four groups of small-scale problems are 2, 3, 3 and 4 .

As described in section 2.2.4, we improve the solving scale and efficiency of the model by fixing $K_{i}$ (the number of storage locations for product $i$ to be placed). As shown in 
Table 1, $\operatorname{SSS}^{K_{i}}$ can obtain the same optimal solutions for 5-8, 6-12 and 8-16 as SSS, and save $43.8 \%, 97.5 \%$ and $92 \%$ of CPU computation time. Moreover, $\operatorname{SSS}^{K_{i}}$ can solve 8-20 which cannot be solved using SSS.

Table 1 Comparison of the different models

\begin{tabular}{ccccc}
\hline $\begin{array}{c}\text { Small-scale } \\
\text { problems }\end{array}$ & & SSS $_{\mathrm{T}}$ & $\mathrm{SSS}$ & $\mathrm{SSS}^{K_{i}}$ \\
\hline \multirow{2}{*}{$\mathbf{5 - 8}$} & Opt & 47.236 & 37.374 & 37.374 \\
& Time & 0.920 & 0.425 & 0.239 \\
\hline \multirow{2}{*}{$\mathbf{6 - 1 2}$} & Opt & 82.386 & 49.291 & 49.291 \\
& Time & 58.914 & 46.837 & 1.183 \\
\hline \multirow{2}{*}{$\mathbf{8 - 1 6}$} & Opt & - & 79.435 & 79.435 \\
& Time & - & 16142.461 & 1290.197 \\
\hline \multirow{2}{*}{$\mathbf{8 - 2 0}$} & Opt & - & - & 72.281 \\
& Time & - & - & 13631.493 \\
\hline
\end{tabular}

"Opt" = the average optimal objective value.

"Time" = the average CPU time.

"_." the instances were not solved.

\subsection{Computational results for the different algorithms}

From the results in Table 1, it can be seen that the model will not be able to solve problems beyond the small-scale ones. In this section, we first use the proposed algorithms to solve the small-scale problems to test their effectiveness and then compare their effectiveness on medium and large-scale problems.

The GA, the basic PSO, the new PSO and the hybrid algorithm are denoted as "GA", "PSO_B", "PSO_X" and "GA+PSO_X", respectively. The parameters are set according to preliminary test results so that they are all at their best performance. For all algorithms, the population size is 200. In GA, crossover and mutation rates are set to 0.9 and 0.15 , respectively, and the maximum number of iterations is 500. In PSO_B, $c_{1}=c_{2}=2, w=0.5, r_{1}$ and $r_{2}$ are random numbers from [0,1], and the maximum number of iterations is 350 . In PSO_X, the maximum number of iterations is 320. In GA+PSO_X, the iteration numbers for the GA part and the PSO_X part are 250 and 150, respectively. The iteration numbers for different algorithms are set such that the computation times of the algorithms on the same problem size are about the same.

Table 2 compares the objective values of the algorithms and models for the small-scale problems. The table shows that the results of the four algorithms are the same as the optimal solutions obtained by the models for 5-8. For 6-12, only PSO_X and GA+PSO_X can obtain optimal solutions. For 8-16 and 8-20, the solutions obtained by the four algorithms are all close to the model solutions. GA+PSO_X gives the best solutions among 
the four algorithms. The gaps between GA+PSO_X and $\operatorname{SSS}^{K_{i}}$ are $1.17 \%$ and $3.64 \%$ for 8-16 and 8-20, respectively.

Table 2. Comparison of different algorithms and models for small-scale problems

\begin{tabular}{cccccccc} 
Small & SSS & SSS $^{K_{i}}$ & GA & PSO_B & PSO_X & GA+PSO_X & Gap \\
\hline $\mathbf{5 - 8}$ & 37.374 & 37.374 & 37.374 & 37.374 & 37.374 & 37.374 & 0 \\
$\mathbf{6 - 1 2}$ & 49.291 & 49.291 & 49.324 & 50.097 & 49.291 & 49.291 & 0 \\
$\mathbf{8 - 1 6}$ & 79.435 & 79.435 & 82.217 & 81.327 & 81.316 & 80.362 & $1.17 \%$ \\
$\mathbf{8 - 2 0}$ & - & 72.281 & 77.585 & 76.117 & 76.141 & 74.911 & $3.64 \%$ \\
"-_" = the instances were not solved. \\
"Gap" = the gap between the model fixing $K_{i}$ and GA+PSO_X.
\end{tabular}

Table 3 gives the CPU computation time results of different algorithms for the medium and large-scale problems. Here, the CPU time is measured in seconds. From the results, we can see that though we set the parameters to expect the same computation time for all algorithms to solve the same problem group, their actual computation times for a group are slightly different. Nevertheless, the differences are not significant and the computing times of all the algorithms increase with problem scale in a similar pattern. The computation time for the problem group with 500 products and 1000 locations is about 10 minutes, indicating that these algorithms can solve larger scale problems.

Table 3 CPU time comparison for medium and large scale problems

\begin{tabular}{ccccc}
\hline Medium & GA & PSO_B & PSO_X & GA+PSO_X \\
\hline $\mathbf{4 0 - 8 0}$ & 5.857 & 6.452 & 5.231 & 5.227 \\
$\mathbf{5 0 - 8 0}$ & 6.607 & 7.151 & 5.649 & 5.636 \\
$\mathbf{5 0 - 1 0 0}$ & 8.623 & 9.233 & 8.208 & 7.711 \\
$\mathbf{6 0 - 1 0 0}$ & 9.853 & 10.365 & 8.406 & 8.340 \\
$\mathbf{1 0 0 - 2 0 0}$ & 30.662 & 29.303 & 29.100 & 27.414 \\
$\mathbf{1 2 0 - 2 0 0}$ & 32.814 & 32.564 & 30.528 & 29.544 \\
\hline & & & & \\
\hline \multirow{2}{*}{$\mathbf{2 a r g e}$} & GA & PSO_B & PSO_X & GA+PSO_X \\
\hline $\mathbf{3 0 0 - 6 0 0}$ & 224.939 & 225.902 & 220.268 & 214.930 \\
$\mathbf{4 0 0 - 8 0 0}$ & 400.464 & 403.014 & 398.550 & 379.142 \\
$\mathbf{5 0 0 - 1 0 0 0}$ & 630.084 & 615.952 & 629.006 & 603.132 \\
\hline
\end{tabular}

To compare the quality of solutions obtained by the algorithms, we first find the minimum among the objective values obtained by all the four algorithms for each instance in a problem group, then compute the relative deviation between the objective value obtained by each algorithm and the minimum objective value, and finally compute the average relative deviation for the ten instances in each problem group. The average relative deviations of all tested algorithms for all problem groups are presented in Table 4. 
Table 4 Objective comparison for medium and large scale problems

\begin{tabular}{ccccc}
\hline Medium & GA & PSO_B & PSO_X & GA+PSO_X \\
\hline $\mathbf{4 0 - 8 0}$ & $7.698 \%$ & $7.494 \%$ & $1.468 \%$ & $\mathbf{0 . 1 0 2 \%}$ \\
$\mathbf{5 0 - 8 0}$ & $7.889 \%$ & $8.064 \%$ & $1.891 \%$ & $\mathbf{0 . 0 3 5 \%}$ \\
$\mathbf{5 0 - 1 0 0}$ & $7.740 \%$ & $7.960 \%$ & $1.285 \%$ & $\mathbf{0 . 0 0 0 \%}$ \\
$\mathbf{6 0 - 1 0 0}$ & $8.721 \%$ & $7.972 \%$ & $1.356 \%$ & $\mathbf{0 . 0 0 0} \%$ \\
$\mathbf{1 0 0 - 2 0 0}$ & $10.048 \%$ & $9.677 \%$ & $1.081 \%$ & $\mathbf{0 . 0 5 0} \%$ \\
$\mathbf{1 2 0 - 2 0 0}$ & $10.143 \%$ & $10.106 \%$ & $2.252 \%$ & $\mathbf{0 . 3 4 4 \%}$ \\
\hline \multicolumn{5}{c}{} \\
\hline \multirow{2}{*}{ Large } & GA & PSO_B & PSO_X & GA+PSO_X \\
\hline $\mathbf{3 0 0 - 6 0 0}$ & $14.021 \%$ & $16.778 \%$ & $3.477 \%$ & $\mathbf{0 . 5 4 0 \%}$ \\
$\mathbf{4 0 0 - 8 0 0}$ & $14.064 \%$ & $18.701 \%$ & $5.088 \%$ & $\mathbf{0 . 5 8 0} \%$ \\
$\mathbf{5 0 0 - 1 0 0 0}$ & $12.950 \%$ & $15.726 \%$ & $3.296 \%$ & $\mathbf{0 . 5 8 6 \%}$ \\
\hline Average & $10.364 \%$ & $11.386 \%$ & $2.355 \%$ & $0.249 \%$ \\
\hline
\end{tabular}

From the results in Table 4, we can see that the average relative deviation of GA+PSO_X is closest to 0 , because its objective values for most instances reached the minimum objective values in the corresponding problem groups. GA and the basic PSO have relatively large average relative deviations indicating that it is difficult for the generic heuristic search procedures to reach close-to-optimal solutions when problem is large. Our proposed new PSO made tremendous improvement over the basic PSO, reducing the overall average relative deviation from $11.386 \%$ to $2.355 \%$. This suggests that velocity and position updating methods we designed considering the problem structure work very well. Combining it with GA, the hybrid algorithm GA+PSO_X makes further improvements.

\section{Conclusions}

In this paper, we first proposed a new scattered storage assignment strategy considering the correlation between products in modern B2C warehouses. By storing several products frequently ordered in one order in nearby locations can reduce the unproductive picking time. A 0-1 integer linear programming model was formulated, and GA and basic PSO algorithm were applied to solve this problem. To improve solution quality, a new PSO algorithm was designed considering the problem characteristic and a hybrid algorithm combining it and GA was proposed. Experimental results demonstrated that the new scattered storage strategy is better than the traditional continuous storage strategy. All the algorithms demonstrated very good performance for small-scale problems. For large scale problems, the solutions obtained by the new PSO and the hybrid algorithm perform much better than the basic algorithms. Future research may be performed to study 
the scattered storage strategy with different objective functions because the distance in the objective is an estimated value in the stage of the storage location assignment rather than the actual distance of pickers. Here, we adopted a straightforward way that minimizing the weighted sum of the shortest distances of all product pairs. We can certainly change the shortest distance for each product pair to other distance. For example, minimize the weighted sum of the average distances of all product pairs.

\section{Acknowledgements}

This research is partly supported by the program of China Scholarships Council (No.201808330034), the National Natural Science Foundation of China (71472081), the Fund for Innovative Research Group of the National Natural Science Foundation of China (71621061), the Major International Joint Research Project of the National Natural Science Foundation of China (71520107004), and the 111 Project (B16009).

\section{References}

Bahrami, Behnam, El-Houssaine Aghezzaf, and Veronique Limere. 2019. "Enhancing the order picking process through a new storage assignment strategy in forward-reserve area." International Journal of Production Research $57 \quad$ (21):6593-614. doi: 10.1080/00207543.2019.1567953.

Bottani, E., M. Cecconi, G. Vignali, and R. Montanari. 2012. "Optimisation of storage allocation in order picking operations through a genetic algorithm." International Journal of Logistics-Research and Applications 15 (2):127-46. doi: 10.1080/13675567.2012.694860.

Boysen, N., R. de Koster, and F. Weidinger. 2019. "Warehousing in the e-commerce era: A survey." European Journal of Operational Research 277 (2):396-411. doi: 10.1016/j.ejor.2018.08.023.

Bozer, Y. A., and J. W. Kile. 2008. "Order batching in walk-and-pick order picking systems." Review of. International Journal of Production Research 46 (7):1887-909. doi: 10.1080/00207540600920850.

Calzavara, Martina, Christoph H. Glock, Eric H. Grosse, and Fabio Sgarbossa. 2019. "An integrated storage assignment method for manual order picking warehouses considering cost, workload and posture." Review of. International Journal of Production Research 57 (8):2392-408. doi: 10.1080/00207543.2018.1518609.

Chan, Felix T. S., and H. K. Chan. 2011. "Improving the productivity of order picking of a manual-pick and multi-level rack distribution warehouse through the implementation of class-based storage." Expert Systems with Applications 38 (3):2686-700. doi: 10.1016/j.eswa.2010.08.058.

Chen, L., A. Langevin, and D. Riopel. 2010. "The storage location assignment and interleaving 
problem in an automated storage/retrieval system with shared storage." International Journal of Production Research 48 (4):991-1011. doi: 10.1080/00207540802506218.

De Koster, M.B.M. and Neuteboom, A.J. 2001. The logistics of supermarket chains. Elsevier, Doetinchem, The Netherlands.

De Koster, R., T. Le-Duc, and K. J. Roodbergen. 2007. "Design and control of warehouse order picking: A literature review." European Journal of Operational Research 182 (2):481-501. doi: 10.1016/j.ejor.2006.07.009.

Fibrianto, Henokh Yernias, and Soondo Hong. 2019. "Dynamic order batching in bucket brigade order picking systems with consecutive batch windows and non-identical pickers." Review of. International Journal of Production Research 57 (20):6552-68. doi: 10.1080/00207543.2019.1567948.

Frazelle, E. A., and G. P. Sharp. 1989. "Correlated assignment strategy can improve any order-picking operation." Industrial Engineering 21 (4):33-7.

Gu, Jinxiang, Marc Goetschalckx, and Leon F. McGinnis. 2010. "Research on warehouse design and performance evaluation: A comprehensive review." European Journal of Operational Research 203 (3):539-49. doi: 10.1016/j.ejor.2009.07.031.

Guan, M.C., and Z.P. Li. 2018. "Genetic algorithm for scattered storage assignment in Kiva mobile fulfillment system." American Journal of Operations Research 08 (06):474-85.

Hausman, W.H., Schwarz, L.B. and Graves, S.C. 1976. Optimal storage assignment in automatic warehousing systems. Management Science, 22, 629-38. Doi: 10.1287/mnsc.22.6.629

Il-Choe, K., and G. Sharp. 2014. "Small Parts Order Picking: Design and Operation."

Kuo, R. J., P. H. Kuo, Y. R. Chen, and F. E. Zulvia. 2016. "Application of metaheuristics-based clustering algorithm to item assignment in a synchronized zone order picking system." Applied Soft Computing 46:143-50. doi: 10.1016/j.asoc.2016.03.012.

Muppani, V. R., and G. K. Adil. 2008. "Efficient formation of storage classes for warehouse storage location assignment: A simulated annealing approach." Omega-International Journal of Management Science 36 (4):609-18. doi: 10.1016/j.omega.2007.01.006.

Pan, J. C. H., P. H. Shih, M. H. Wu, and J. H. Lin. 2015. "A storage assignment heuristic method based on genetic algorithm for a pick-and-pass warehousing system." Computers \& Industrial Engineering 81:1-13. doi: 10.1016/j.cie.2014.12.010.

Pang, K. W., and H. L. Chan. 2017. "Data mining-based algorithm for storage location assignment in a randomised warehouse." International Journal of Production Research 55 (14):4035-52. doi: 10.1080/00207543.2016.1244615.

Roodbergen, K. J., and I. F. A. Vis. 2009. "A survey of literature on automated storage and retrieval systems." European Journal of Operational Research 194 (2):343-62. doi: 10.1016/j.ejor.2008.01.038.

Ruben, R. A., and F. R. Jacobs. 1999. "Batch construction heuristics and storage assignment strategies for walk/ride and pick systems." Management Science 45 (4):575-96. doi: 10.1287/mnsc.45.4.575.

Tang, L. X., Y. Dong, and J. Y. Liu. 2015. "Differential Evolution With an Individual-Dependent Mechanism." IEEE Transactions on Evolutionary Computation 19 (4):560-74. doi: 
$10.1109 /$ tevc.2014.2360890.

Tang, L. X., D. F. Sun, and J. Y. Liu. 2016. "Integrated storage space allocation and ship scheduling problem in bulk cargo terminals." IIE Transactions 48 (5):428-39. doi: 10.1080/0740817x.2015.1063791.

Tang, L.X., F. Li, and Z.L. Chen. 2019. "Integrated scheduling of production and two-stage delivery of make-to-order products: offline and online algorithms." INFORMS Journal on Computing 31 (3):411-632. doi: 10.1287/ijoc.2018.0842.

Weidinger, F. 2018. "Picker routing in rectangular mixed shelves warehouses." Computers \& Operations Research 95:139-50. doi: 10.1016/j.cor.2018.03.012.

Weidinger, F., and N. Boysen. 2018. "Scattered storage: how to distribute stock keeping units all around a mixed-shelves warehouse." Transportation Science 52 (6):1412-27. doi: $10.1287 /$ trsc.2017.0779.

Weidinger, F., N. Boysen, and M. Schneider. 2019. "Picker routing in the mixed-shelves warehouses of e-commerce retailers." European Journal of Operational Research 274 (2):501-15. doi: 10.1016/j.ejor.2018.10.021.

Xiang, X., C. C. Liu, and L. X. Miao. 2018. "Storage assignment and order batching problem in Kiva mobile fulfilment system." Engineering Optimization 50 (11):1941-62. doi: 10.1080/0305215x.2017.1419346.

Xiao, J., and L. Zheng. 2010. "A correlated storage location assignment problem in a single-block-multi-aisles warehouse considering BOM information." International Journal of Production Research 48 (5):1321-38. doi: 10.1080/00207540802555736.

Yang, L. Q., Y. Zheng, Y. Z. Xu, and Y. J. Bai. 2017. "Research on location assignment model of intelligent warehouse with RFID and improved particle swarm optimization algorithm", 2017 International Conference on Computer Systems, Electronics and Control :1262-1266.

Yang, P., L. X. Miao, Z. J. Xue, and B. Ye. 2015. "Variable neighborhood search heuristic for storage location assignment and storage/retrieval scheduling under shared storage in multi-shuttle automated storage/retrieval systems." Transportation Research Part E-Logistics and Transportation Review 79:164-77. doi: 10.1016/j.tre.2015.04.009.

Yu, Yugang, and Rene B. M. de Koster. 2013. "On the suboptimality of full turnover-based storage." International Journal of Production Research $51 \quad$ (6):1635-47. doi: 10.1080/00207543.2011.654012.

Zhao, N., M. J. Xia, C. Mi, Z. C. Bian, and J. Jin. 2015. "Simulation-Based Optimization for Storage Allocation Problem of Outbound Containers in Automated Container Terminals." Mathematical Problems in Engineering 2015 (1):548762. 